Journal of Statistical Physics, Vol. 36, Nos. 5/6, 1984

\title{
Application of Fractal Concepts to Polymer Statistics and to Anomalous Transport in Randomly Porous Media
}

\author{
H. Eugene Stanley ${ }^{1,2}$
}

\begin{abstract}
Received
We consider the application of fractal concepts to polymer statistics and to anomalous transport in randomly porous media. It is found that answers to interesting physics questions can be expressed in terms of several new fractal dimensions (in addition to "the" fractal dimension $d_{f}$ ): (1) $d_{f}^{B B}$, the fractal dimension of the backbone, arises in connection with electric current flow, (2) $d_{\text {red }}$, the fractal dimension of the singly connected bonds in the backbone, arises in connection with its equivalence to the thermal scaling power, (3) $d_{E}$, the fractal dimension of the of the elastic backbone, (4) $d_{u}$, the fractal dimension of the unscreened perimeter, arises in connection with the viscosity singularity at the gelation threshold, $(5) d_{\min }$ the fractal dimension of the minimum path (or "chemical distance") between two sites, arises in connection with the Aharony-Stauffer conjecture, (6) $d_{w}$, the fractal dimension of a random walk, (7) $d_{G}$, the fractal dimension of growth sites that arise as a random walk creates a cluster. Relations among these fractal dimensions are discussed, some of which can be proved and others of which are conjectures whose validity has been established only in certain limiting cases.
\end{abstract}

There is an underground joke circulating that the three great diseases of the latter part of the twentieth century will be remembered as being Herpes, AIDS, and fractals. Indeed, fractals has at least one earmark of a great disease: it can spread. While just a few years ago, one heard of only a single fractal dimensionality, $d_{f}$, nowadays one hears of at least nine separate fractal dimensions. In addition, it is sometimes useful to form the ratio of two fractal dimensions and thereby to form what Toulouse calls an "intrinsic" fractal dimension... analogous perhaps to the family of crossover or "gap" exponents obtained when one formally takes the ratio of scaling

\footnotetext{
${ }^{1}$ Center for Polymer Studies and Department of Physics, Boston University, Boston, Massachusetts 02215.

${ }^{2}$ Supported in part at the Center for Polymer Studies by grants from ONR and NSF.
} 
powers. ${ }^{(1)}$ If there are nine extrinsic fractal dimensions, then there are potentially $\left(\begin{array}{l}9 \\ 2\end{array}\right)$ possible intrinsic fractal dimensions. Let us hope that our current situation is analogous to that in critical phenomena just before Widom and Kadanoff put forth the scaling conjecture. There were, in the early 1960s, more exponents in use than letters in the Greek alphabet: in one stroke the scaling conjecture reduced this surfeit to a mere two independent exponents.

The purpose of this talk is to introduce some of these newer fractal concepts, with particular emphasis on those that seem to be of some utility in polymer statistics and in describing anomalous transport in randomly porous media. We shall also discuss several recently discovered relations among the various fractal dimensions.

We must take care to distinguish the utility of regular geometric fractals and irregular, random, "statistical" fractals. ${ }^{(2)}$ For regular "geometric" fractals,

$$
[\text { mass }]_{\text {tot }}=L^{d_{f}} \quad \text { ("exactly") }
$$

Here $M$ denotes the number of objects in the fractal (its "mass") and $L$ denotes the length scale under which it is examined.

For irregular (random) or "statistical" fractals, the symbols in (1a) are replaced by their mean values: $M$ denotes the mean mass and $L$ denotes the rms radius of gyration (or caliper length - one seems to get the same answer regardless ${ }^{3}$ ). Thus we write

$$
[\text { mass }]_{\text {tot }} \sim L^{d_{f}} \quad \text { ("asymptotically") }
$$

For some statistical fractals, one can compute $d_{f}$ exactly; for others, we use a computer. In practice, we must average over thousands (sometimes even millions) of realizations in order to compute accurate estimates of $d_{f}$.

Before formally beginning, I should apologize to those whose work I have omitted, and acknowledge my colleagues whose work I have emphasized; these include Z. Djordjevic, S. Havlin, H. J. Herrmann, D. Hong, N. Jan, F. Leyvraz, I. Majid, P. Meakin, and especially A. Coniglio. It is also a pleasure to thank the conference organizers for having invited me.

\section{INTRODUCTION}

The basic phenomenon of interest in porous media is connectivity; e.g., in order that oil flow to the surface of the earth, there must be some

${ }^{3}$ For example, for the lattice animal model of randomly branched polymers, the fractal dimension was independently calculated using two different quantities as the effective "length," the caliper length (Reference $3 a$ ) and the rms radius of gyration (Reference $3 b$ ). The same answer was obtained in both calculations, to within the quoted accuracy. 

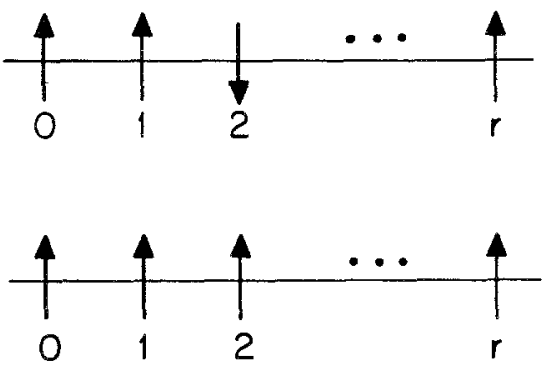

(a) Pair correlation function

(b) Pair correctedness

Fig. 1. Analogous functions for the spin problem and the connectivity problem: (a) The pair correlation function measures the probability a spin at $r$ is pointing in the same direction as a spin at the origin, and (b) the pair connectedness measures the probability that there is a connected path of "up" spins between the spin at $r$ and the spin at the origin.

connected path. Let me emphasize that connectivity questions are quite different from those we address in ordinary thermal critical phenomena. For example, in the Ising model we study the spin correlation function, which is related to the probability that two objects ("spins") separated a distance $r$ are in the same state. ${ }^{(1)}$ Here we will study the pair connectedness function, the probability that two objects separated a distance $r$ are in the same state and also are connected by some path between them, all of whose objects are in the same state (Fig. 1). ${ }^{(4)}$

There are two reasons we care about transport in fractals. One is that problems involving connectivity are extremely deep and subtle, and hence seduce our theoretical interest. A second is that there is a wide range of systems in Nature for which the essential physics is connectivity. ${ }^{4,(6-18)}$ One such example, porous earth, was just mentioned. A second but related example is randomly branched structures like polymers-you could make such a structure by fabricating a plaster cast of the ramified pore structure in porous earth. The list of additional applications ranges from the study of the spread of disease to the design of lightweight mechanical materials.

When man went to the moon, there no really big physics surprises. This is because the laws of physics that work on Earth also work on the moon. To our surprise, we are finding that this is not the case for randomly porous materials. Specifically, Fick's law of diffusion states that

$$
\left\langle r^{2}\right\rangle=N_{w} \quad(d=1,2,3, \ldots)
$$

where $\left\langle r^{2}\right\rangle$ is the rms displacement of a random walk of $N_{w}$ steps. If the random walker is obliged to be on a fractal, then he travels much less far in

\footnotetext{
${ }^{4}$ See, for example, Reference 5, which reports the proceedings of an International Topical Conference on this subject.
} 


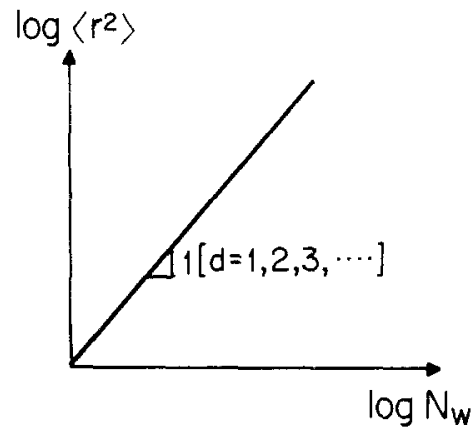

(a) Euclidean lattice

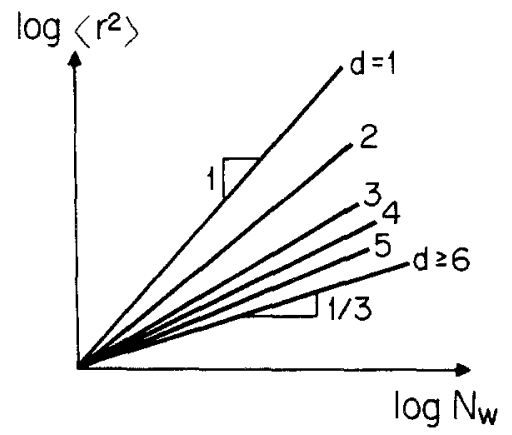

(b) Percolation cluster at $p=p_{c}(d)$

Fig. 2. Schematic illustration of the dependence of the mean square displacement on the number of steps of a random walk on (a) a Euclidean lattice, and (b) a percolation cluster at the percolation threshold.

the same time. This could reflect itself in a prefactor in (2a), but in fact the entire law is changed-one finds (Fig. 2) ${ }^{(19)}$

$$
\left\langle r^{2}\right\rangle \sim\left(N_{w}\right)^{x} \quad(x=x(d) \leqslant 1)
$$

One may think of the random walker leaving behind a little piece of bread at each step so that it is possible to define the fractal dimension $d_{w}$ of walk through

$$
[\text { mass }]_{\text {walk }} \sim L^{d_{w}} \quad\left(d_{w}=d_{w}(d) \geqslant 2\right),
$$

where [mass $]_{\text {walk }}$ is the total number of steps (the total "mass" of bread), and $L$ is the rms displacement (or "range") of the walker.

\section{REGULAR GEOMETRIC FRACTALS}

Consider the structure shown in Fig. 3a. This is an example of a regular or "geometric" fractal sometimes called the Sierpinski gasket; its dual has recently been shown to be the Bethe lattice. ${ }^{(20)}$ At first sight there is nothing particularly intriguing about this structure-one iterates a simple rule of cutting out the center of each triangle until one gets tired. However, this structure has a remarkable property: Its density depends on the length scale $L$ with which the structure is probed. To see this, suppose we examine one of the smallest triangles of Fig. $3 \mathrm{a}$. Let us define the edge of this triangle to have length $L=1$, and the entire triangle to have mass $M=1$. The density on this "scale" is then $\rho=M / L^{2}=1$. Now consider the next smallest 

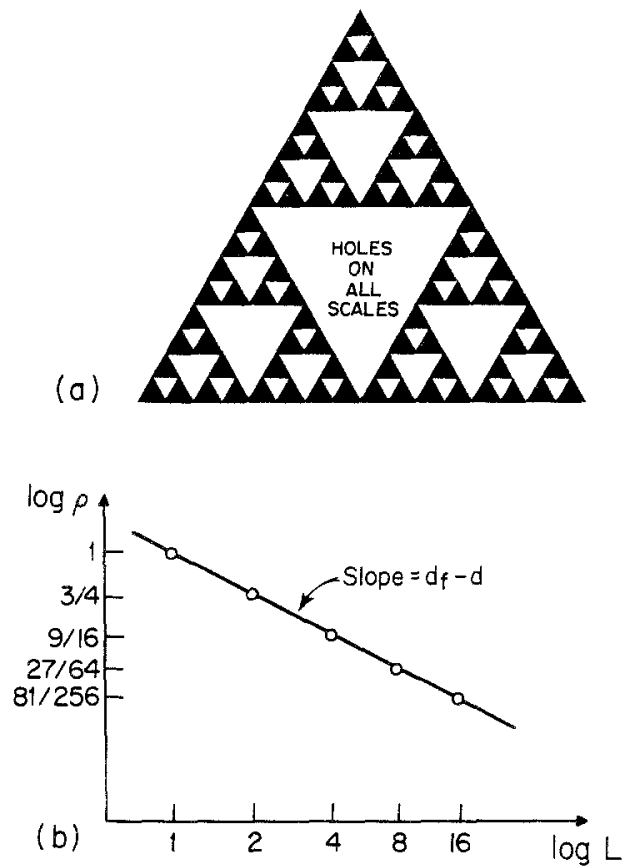

Fig. 3. A Sierpinski gasket that is self-similar on all length scales. The density decreases with the length scale, in a fashion governed by the codimension $d_{f}-d$.

triangles. Clearly $L=2$, but $M=3$ (not 4 ) because of the hole. Thus $\rho=3 / 4$. The next larger triangles have $L=4$ and $M=9$ (not 16), so $\rho=9 / 16$. Finally, the largest triangle shown (the entire structure) has $L=8$ and $M=27$ (not 64 ), so that $\rho=27 / 64$. Clearly the density is decreasing with $L$, unlike a normal solid object. Figure $3 b$ shows a $\log -\log$ plot of $\rho$ vs. $L$. From (1a), we expect $\rho \sim L^{d_{f^{-}} d}$ so that the slope of Fig. $3 \mathrm{~b}$ should be $d_{f}-2$. A quick calculation, based on the first two points, shows that the "codimension" $d_{f}-d$ is given by

$$
d_{f}-d=[\ln 1-\ln (3 / 4)] /(\ln 2-\ln 1)=(\ln 3 / \ln 2)-2
$$

so that $d_{f}=(\ln 3 / \ln 2)=1.6$. Generalizing to $d>2$, one finds

$$
d_{f}(\text { Sierpinski gasket })=\ln (d+1) /(\ln 2)
$$

One can calculate $d_{w}(d)$ exactly for the gasket, with the result

$$
d_{w}(\text { Sierpinski gasket })=\ln (d+3) /(\ln 2)
$$

so that $d_{w}=d_{w}(d) \geqslant 2$, as noted in Eq. (2c). 
We conclude this section with two remarks.

(i) Note that a necessary condition for linearity of Fig. 3b is that there be "holes" of all sizes in the fractal object of Fig. 3a. Suppose, e.g., that our object had holes of only the smallest two sizes. Then the density would decrease as shown in Fig. 4 from 1 to $3 / 4$ to 9/16, but would "stick" at 9/16 thereafter. The behavior for values of $L$ larger than 4 would correspond to simply "patching together" smaller fractal objects to form a macroscopically homogeneous structure. We say that the value $L=4$ represents a crossover from self-similar fractal behavior for $L>4$. Since for sufficiently large $L$, the slope of Fig. $4 \mathrm{~b}$ is zero we conclude that the material is simply twodimensional at large scales even though it is 1.6-dimensional at very small scales. A truckload of donuts is 3-dimensional, no matter what hole distribution the individual donuts have. We can safely predict that if we double the linear dimension of the cargo space that the mass of the load will increase by a factor of 8 .

(ii) Note that the mass $M$ in Fig. 3 is a homogeneous function of $L$, and that $d_{f}$ is the degree of homogeneity. That is, for all values of $\lambda$,

$$
M(\lambda L)=\lambda^{d_{f}} M(L)
$$

(a)
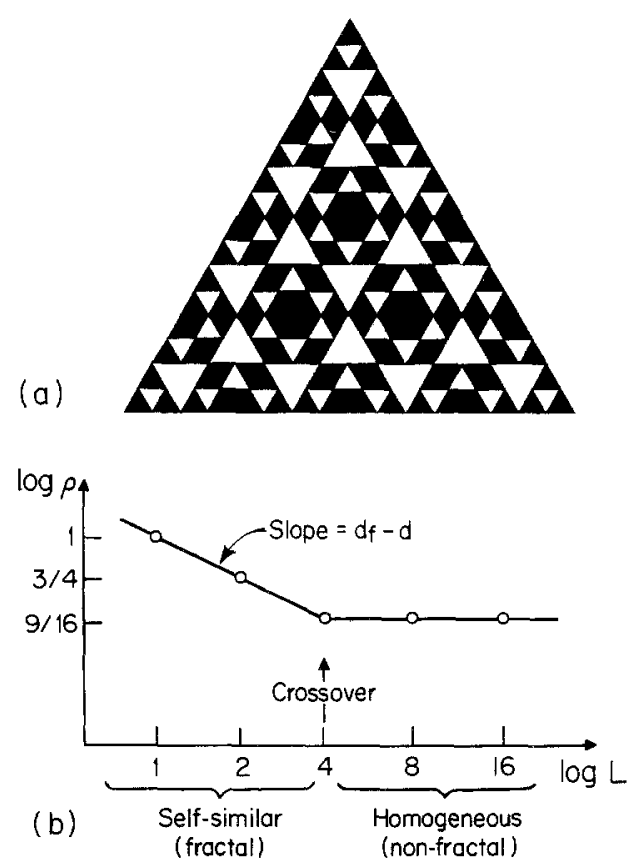

Fig. 4. A Sierpinski gasket that is self-similar (fractal) on small length scales, but becomes homogeneous (nonfractal) on large length scales. 
Equation (4) is a hallmark in critical phenomena. "Functional equations" such as (4) play a role in critical phenomena analogous to that played by algebraic equations in conventional physics. While the solution of an algebraic equation is a number, the solution of a functional equation is a function. For example, the solution of Eq. (4) is the function $M(L) \sim L^{d_{f}}$, which can be obtained perhaps most simply by setting $\lambda=1 / L$ in (4).

\section{STATISTICAL (RANDOM) FRACTALS}

Now we are ready to describe the extension of the fractal concept to random fractals, such as the interior space of a porous rock. At first sight it might seem impossible to make any concrete predictions (like the values of $d_{f}$ or $d_{w}$ ) for a structure that is completely random. Moreover, it might seem impossible to fulfill the requirement that there be "holes of all sizes."

\subsection{Fractal Dimension of Entire Cluster and the Field Scaling Power}

Consider the paradigm problem of site percolation. ${ }^{(4)}$ To describe this problem, imagine a chessboard that is infinite in spatial extent. Imagine also that one randomly occupies a fraction $p$ of the squares by pawns. When $p$ is small, most pawns are isolated. However, as $p$ increases one begins to see "clusters" of two, three,... pawns. Naively, one might expect that as $p$ increases we would find more large clusters than small clusters. This is not the case. Rather, the cluster size distribution function, giving the probability $P_{s}$ that a randomly selected site belongs to an $s$-site cluster, is a monotonically decreasing function of $s$ for all values of $p$ (Fig. 5a). In fact, a $\log -\log$ plot of $P_{s}$ vs. $s$ is linear with slope $-d / d_{f}$ for all values of $s$ up to a characteristic value $s^{*} \sim \xi^{d_{f}}$, where $\xi$ is the pair connectedness length that characterizes the decay with distance of the function that gives the probability of a site at random $r$ to be connected to a site at the origin.

As $p$ continues to increase beyond the value 0.50 we find that the cluster size distribution continues to be monotonic, extending to larger and larger values of $s$. Finally, at a critical value $p=p_{c}$, termed the percolation threshold, there appears in addition to the archipelago of finite clusters a single cluster that is infinite in spatial extent. In the 1977 paper that introduced fractals into percolation, ${ }^{(21)}$ it was noted that one reason for the possible interest in $d_{f}$ is that it is equal to the magnetic scaling power $y_{h}$,

$$
y_{h}=d_{f}
$$

Thus fractals provide a geometrical interpretation of what was previously an abstract mathematical quantity! 

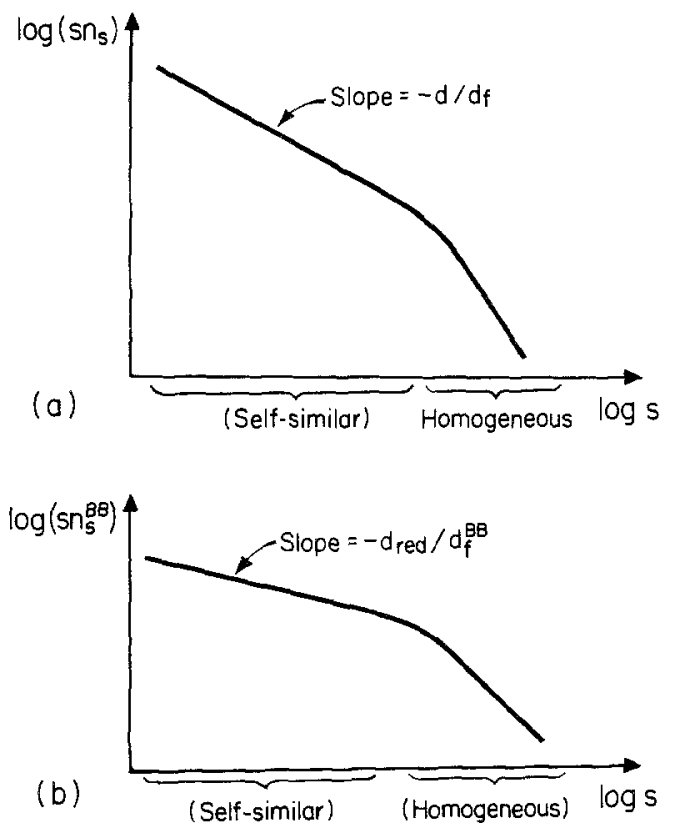

Fig. 5. The dependence on $s$ of $P_{s}=s n_{s}$, the probability that a randomly chosen site belongs to (a) an $s$-site cluster, or (b) an $s$-site "blob" in the percolation backbone. The value of $p$ chosen is slightly below $p_{c}$, so there is a crossover at a large but finite value of $s$ between self-similar behavior and homogeneous behavior, analogous to that shown in Fig. 4 for the Sierpinski gasket.

\subsection{Fractal Dimension of Red Bonds and the Thermal Scaling Power ("Links")}

Is there an analogous geometrical interpretation of the thermal scaling power $y_{T}$ in percolation? A second fractal quantity was introduced in the 1977 paper in connection with the observation that the incipient infinite cluster in percolation consists of multiply connected "blobs" joined by singly connected "links" (Fig. 6). Pike has made a computer simulation of this links/blobs decomposition, published in color in Physics Today, May 1983 - the links are in red and blobs in blue, with the dangling ends in yellow. ${ }^{(22)}$ One defines $d_{\text {red }}$ through

$$
[\text { mass }]_{\text {red }} \sim(\text { length })^{d_{\text {red }}}
$$

with similar definitions for the blue and yellow. Coniglio proved rigorously that for all $d$,

$$
y_{T}=d_{\mathrm{red}}
$$




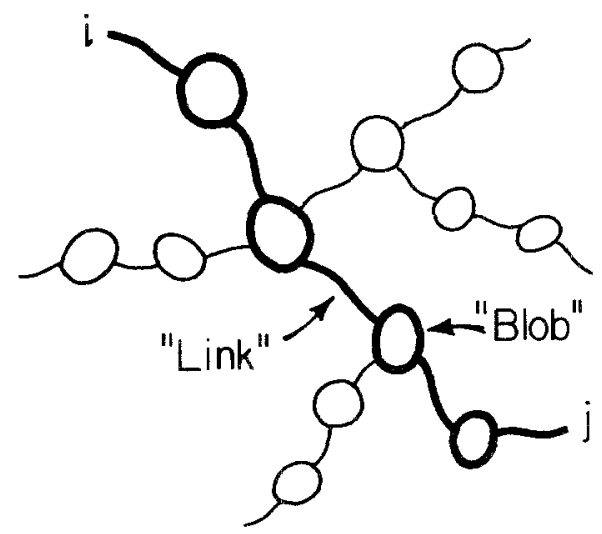

Fig. 6. Typical percolation cluster just below the percolation threshold, indicating backbone bonds (heavy line) and dangling ends (light lines); both are similar in that they are composed of "links and blobs." After Reference 32.

which had been found numerically for $d=2 .^{(23 a-c)}$ Thus both scaling powers in percolation are directly related to geometrical parameters. ${ }^{5}$

\subsection{Fractal Dimension of the Backbone}

Very recently Herrmann and the speaker ${ }^{(24)}$ have initiated a program to study the distribution of elements in the backbone in the percolation cluster. We find that the backbone is made up of singly connected sites and multiplyconnected blobs (Fig. 6). The blobs come in all sizes $s$ (with $s=1$ corresponding to singly connected sites), so it is natural to enquire about the "blob size distribution function." We find that this function obeys a decay law (Fig. 5b) analogous to that found for the full percolation cluster, except that the role of $d$ is played by $d_{\text {red }}$, the fractal dimension of the singly connected sites. Moreover, we find that the distribution of length of the segments of singly connected bonds is exponential, and the same is true for the distribution of length of segments of 2-site blobs, 3-site blobs, and so forth. Thus the backbone of a percolation cluster has a structure identical to what would be obtained if a blind monkey were to choose "building blocks" one-by-one from the probability distribution law of Fig. $5 \mathrm{~b}$ and assemble these building blocks in a linear chain. Thus the backbone of the incipient infinite cluster through which oil first passes has a very simple and elegant structure!

\footnotetext{
"It appears that Coniglio's lemma holds "term-by-term" in the graphical expansion used in exact enumeration methods, as noted in Reference $23 \mathrm{~d}$ and proved in $23 \mathrm{e}$.
} 
Having mentioned the decomposition ${ }^{(21)}$ of the backbone of the incipient infinite cluster into blue blobs and red links, it is natural to consider the fractal dimension of the backbone itself. Thus we define

$$
[\text { mass }]_{\text {backbone }} \sim(\text { length })_{f}^{d_{f}^{\mathrm{BB}}}
$$

Why do we care about the backbone? Suppose we attach the terminals of a battery to two points of a percolation cluster. Then it is the backbone bonds that will carry current, and not the dangling ends. Thus we expect that the backbone will be related to the behavior of the electrical resistance.

\subsection{Fractal Dimension of a Random Walk}

To see the relation directly, we must utilize the Einstein relation: the electrical conductivity is proportional to the time rate of change of $\left\langle r^{2}\right\rangle$, the mean square displacement of a random walker. Now the fractal dimension of a random walk is defined by imagining that the random walk is a free flight polymer chain (with no "excluded volume"). Then the mass of the chain is equal to the number of steps in the random walk, while the length of the walk is the rms displacement $\left\langle r^{2}\right\rangle^{1 / 2}$. Hence we can write, as in (2c), [mass $]_{\text {walk }} \sim(\text { length })^{d_{w}}$. Here $d_{w}$ is called the fractal dimension of the walk. For a nonfractal, $d_{w}=2$ regardless of $d$, while for the fractals studied at this conference $d_{w}$ is a strong function of $d$ and is always larger than two. For example, for percolation clusters $d_{w}$ increases from roughly $91 / 32 \cong 2.85$ for $d=2$ (a conjecture made in 1982 by Alexander and Orbach and probably accurate to at least $2 \%-3 \%$ ) to 6 for $d>6 .{ }^{(25-31)}$ For the percolation backbone, which is much less "ramified," $d_{w}$ increases from roughly 2.77 for $d=2$ to 4 for $d>6 .{ }^{(32)}$ A random walk on a Witten-Sander fractal is shown ${ }^{(30)}$ in Fig. 7.

From the definition (2), a one-line derivation using the Einstein relation shows that physical quantity, the resistance exponent $\tilde{\zeta}$, is given by the difference between two fractal dimensions, ${ }^{(25-31)}$

$$
\tilde{\zeta}=d_{w}-d_{f}
$$

Thus a physical quantity which is normally thought to involve the complex physics of Kirchoffs laws is found to be simply given by the difference between two fractal dimensions. Now the Einstein relation holds even after the dangling ends are chopped off. Hence one can apply the same one-line derivation to the backbone, with the result ${ }^{(32)}$

$$
\tilde{\zeta}=d_{w}^{\mathrm{BB}}-d_{f}^{\mathrm{BB}}
$$




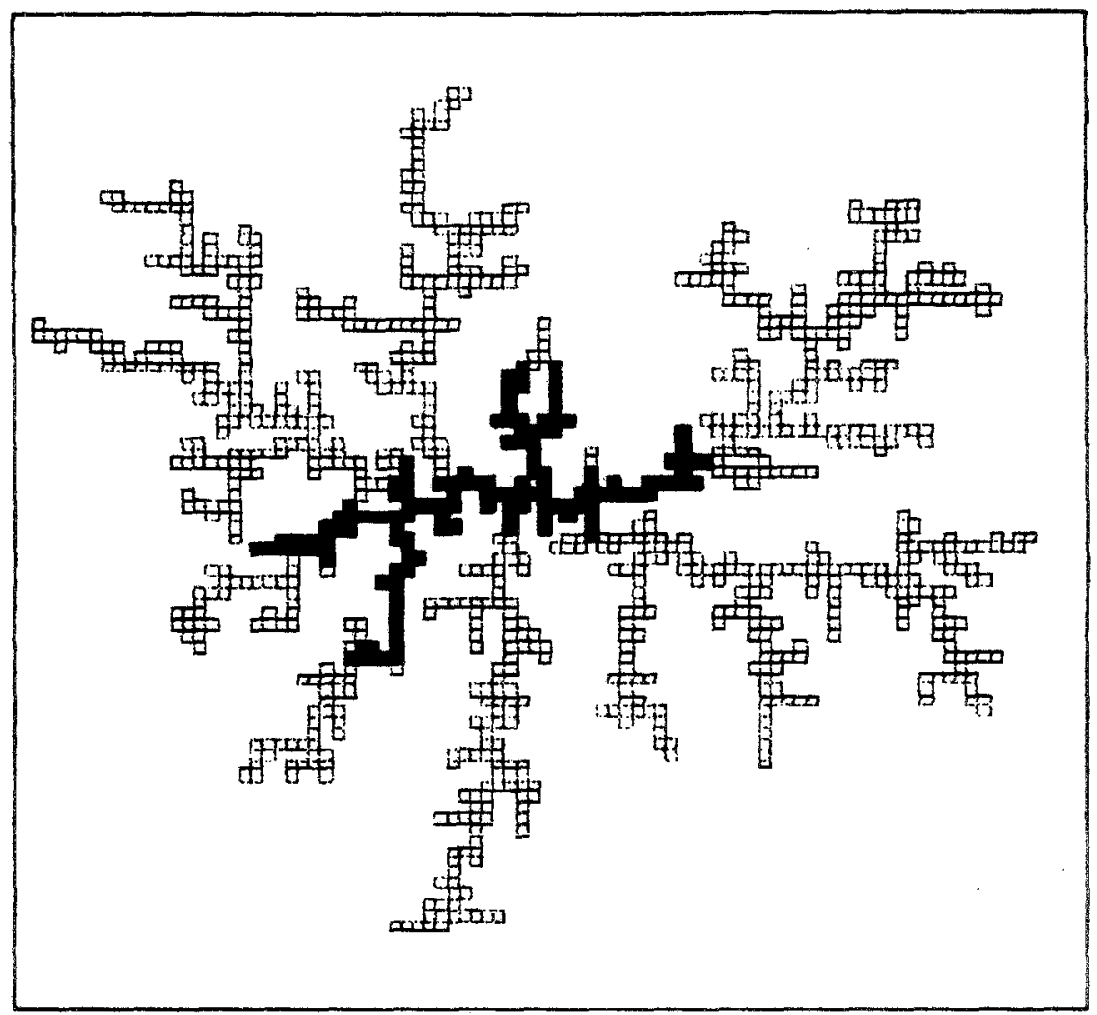

Fig. 7. A random walk of 2500 steps on a fractal with 1000 sites (a small aggregate). The sites visited by the walk are indicated by heavy lines. (From Ref. 30.)

Combining (4a) and (4b), we have a relation between the backbone properties and the properties of the full cluster, ${ }^{(32)}$

$$
d_{w}-d_{f}=d_{w}^{\mathrm{BB}}-d_{f}^{\mathrm{BB}}
$$

\subsection{Fractal Dimension of Growth Sites: The "Order Parameter" of Dynamics?}

This relation between the backbone and the full cluster motivates a search for a fundamental geometric interpretation of the basic dynamic process itself. Our own group's effort along these lines has focussed to a large extent on the Leyvraz/Stanley "growth sites."(3i) These are defined as follows. Imagine that we generate a percolation cluster by dropping an ant onto a square lattice at $t=1$, letting the ant "generate the percolation cluster as it moves." The four neighboring sites are called growth sites because the 
ant has the potential of "growing" his territory by moving there. Now we give the ant two coins. The first has four sites: $N, E, S$, and $W$. The second has two sides, occupied and blocked, and is weighted to come up "blocked" $41 \%$ of the time. Thus at each time step the ant flips the two coins in succession and moves if possible. The number of $G$ sites clearly increases with time. Moreover, as Rammal and Toulouse ${ }^{(28)}$ pointed out, there is a simple relation between the total number of sites visited by the ant and the total number of $G$ sites.

$$
(d / d N) M_{\mathrm{tot}}=M_{G} / M_{\mathrm{tot}}
$$

where $N=[\text { mass }]_{\text {walk }}$ is the total number of steps, and

$$
M_{G}=[\text { mass }]_{G \text { sites }} \sim(\text { length })^{d_{G}}
$$

which serves to define the fractal dimension $d_{G}$. Substituting (1), (2), and (10) into (6) we find

$$
d_{G}=2 d_{f}-d_{w}
$$

The Alexander-Orbach conjecture (AO) that $d_{w}=(3 / 2) d_{f}=91 / 32$ predicts that $d_{G}=(1 / 2) d_{f}=91 / 96 \cong 0.9479$, while the Aharony-Stauffer conjecture (AS) ${ }^{(33)}$ that $d_{w}=d_{f}+1$ predicts that $d_{G}=d_{f}-1=43 / 48 \cong 0.8958$, about $5 \%$ smaller. Coniglio and Stanley ${ }^{(34)}$ —and independently AS-argued that the critical dimension for dynamic phenomena was $d_{f}=2$ (NOT $d=2$ ); indeed, both predictions become identical at $d_{f}=2$. Geometrically, the AS prediction corresponds to the assumption that the $G$ sites are localized on the Mandelbrot perimeter-i.e., in a ring that does not increase in width as the fractal grows. This is being tested now for percolation fractals and preliminary results can be discussed privately. Such a test would be easier for fractals with lower $d_{f}$, and to this end much attention recently has focussed on a family of fractals studied extensively by Meakin, Leyvraz, and Stanley. ${ }^{(35)}$ Here it appears that one can continuously tune the fractal dimension and more easily test conjectures like those mentioned above which are difficult to test on percolation since $d_{f}$ is so close to 2 .

\subsection{The Fractal Dimension of the Chemical ("Minimum") Path}

Of relevance in a polymer problem is not only the "air"distance between two points (this is the length $L$ ), but also the "cowpath" connecting these two points (Fig. 8). This cowpath was called the minimum path in the early studies of Pike and Stanley for $d=2^{(36)}$ and series studies for $d=2,3$, 4,5 , the renormalization group studies for $d=2$ of Hong and Stanley, ${ }^{(37)}$ 


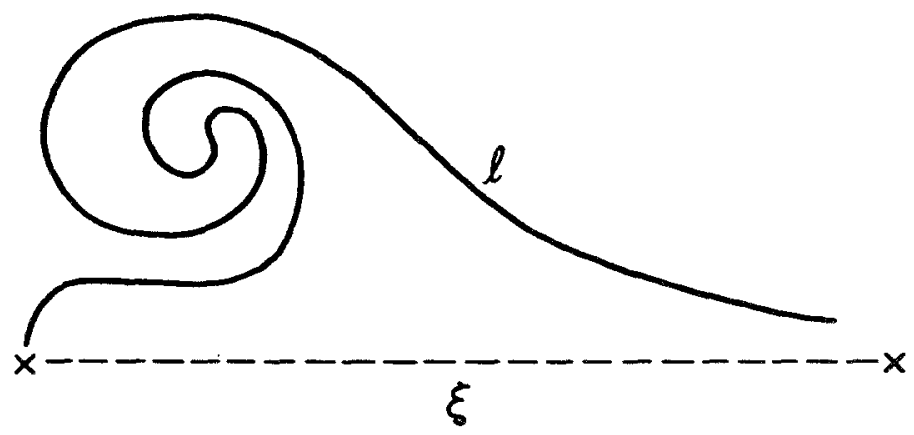

Fig. 8. Schematic illustration of the difference between the "airline distance" between two points and the "cowpath distance" or "chemical length" $\ell$ between the same two points. The exponent $d_{\min }$ characterizes the dependence of $\ell$ on $\xi$. If we count all the sites within a chemical distance $\ell$ of a given site, then this "mass" scales as $\ell^{d_{l}}$, where $d_{l}=d_{f} / d_{\mathrm{n}}$ is the ratio of two fractal dimensions--an intrinsic fractal dimension.

and the rigorous results of Coniglio. ${ }^{(38)}$ The mass of bonds in this minimum path is larger than the mass of bonds would be in an "airline" route, and in fact scales with $L$ with an exponent distinctly larger than one. Thus we can define the fractal dimension $d_{\min }$ by

$$
[\text { mass }]_{\text {min path }} \sim(\text { length })^{d_{\text {min }}}
$$

Alexander has suggested that one think of this minimum path between two sites as the "chemical distance" separating them, and Havlin and collaborators ${ }^{(39-42)}$ have studied the variation of the total cluster mass within a chemical distance $l=M_{\text {min path }}$. Clearly

$$
[\text { mass }]_{\text {tot }} \sim(\text { length })^{d_{f}} \sim l^{d_{f} / d_{\text {min }}}=l^{d_{l}}
$$

and this "intrinsic" exponent $d_{l}$ is termed the chemical dimension. Toulouse has suggested that $d_{l}$ be called the "spreading dimension" since it measures the number of trees felled by a forest fir ${ }^{(44)}$ and Herrmann and his colleagues have devised an efficient algorithm for "burning" a cluster. ${ }^{(45)}$ In any case, there are now several groups measuring $d_{\min }$ (or, equivalently, $d_{l}$ ) and the results are in good agreement. A possible application even more intriguing than forest fires is the work of Ritzenberg and Cohen in modeling cardiac phenomena. ${ }^{(10)}$

The minimum path between two points is the same regardless of whether the dangling ends have been decapitated, so that $d_{\min }$ is the same for the backbone and the full cluster. Hence the analog of $(5 c)$ is

$$
d_{f} / d_{l}=d_{f}^{\mathrm{BB}} / d_{l}^{\mathrm{BB}}
$$




\subsection{Fractal Dimension of the Elastic Backbone}

The backbone of a percolation cluster is sometimes defined as the union of all self-avoiding random walks between two points. The elastic backbone is defined as the union of all minimum paths between two points. ${ }^{(45)}$ Thus the only blobs in the elastic backbone are those that have the same "stress" in all members, so the elastic backbone might represent the essential physics of elasticity phenomena when one neglects the contribution from bond angles (Fig. 9).

Although the blobs in the elastic backbone might at first sight appear to be rare and small, they in fact seem to occur in all sizes. Hence one finds

$$
[\text { mass }]_{\text {elastic в B }} \sim(\text { length })^{d_{E}}
$$

with $d_{E}>d_{\min }$. Herrmann and his collaborators have measured $d_{E}$ for $d=2,3{ }^{(45)}$

\subsection{Fractal Dimension of the Unscreened Perimeter}

Perhaps the most dramatic example of the main point of this lecture is the example that we have saved for last: The fractal dimension $d_{u}$ characterizing the mass of the unscreened perimeter,

$$
[\text { mass }]_{\text {unscreened perimeter }} \sim(\text { length })^{d_{u}}
$$

This quantity arises naturally in describing the electrical conductivity of a random network made of superconducting bonds. Just below the percolation threshold, this problem can be modeled by the de Gennes
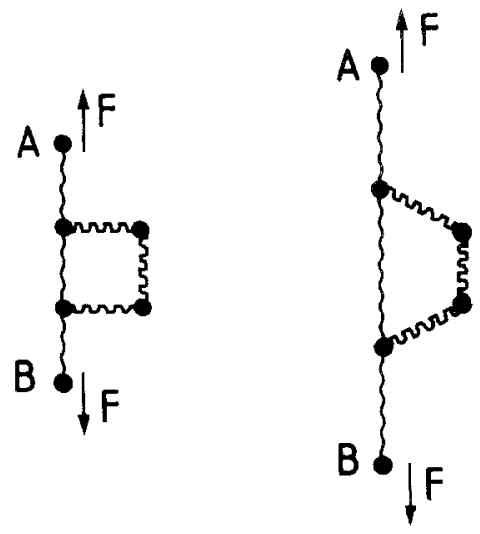

a
Fig. 9. Supposing that the angles of the object of springs shown in (a) can change freely, we show in (b) the elongation of the object due to the application of the force $F$ at $A$ and $B$. (After Ref. 45.) 
termite. ${ }^{(46)}$ The termite, unlike the ant, is free to move off and on the cluster. When off the cluster, it is on normal bonds and hence undergoes a normal random walk. When on the cluster it moves in accord with the superconducting nature of the cluster. Coniglio and Stanley ${ }^{(34)}$ have recently found that the unscreened perimeter of a cluster governs the essential physics of this problem, since the termite can enter and leave a cluster only though this subset of the total perimeter. They obtain a remarkably simple and physically revealing formula

$$
\tilde{\zeta}_{s}=d_{u}
$$

for the exponent governing the divergence of the conductivity $\tilde{\zeta}_{s}$, which is the analog of $\tilde{\zeta}$ defined above for the random resistor network. Moreover, they relate $d_{u}$ to the fractal dimension $d_{P}$ of the projectile $\left(d_{P}=2\right.$ for a random walk or termite) through the simple expression

$$
d_{u}=\left(d_{f}-1\right)+\left(d-d_{f}\right) / d_{P}
$$

The first term in (18) corresponds to the "Mandelbrot perimeter": it gives the exponent that governs how scales the number of sites that are cut by a hypersphere intersecting the fractal. The second term is the increase necessary to account for the fact that there is penetration of the fractal; it is proportional, therefore, to the codimension $d-d_{f}$ and inversely proportional to the fractal dimension of the projectile. Note that in the limiting case $d_{p}=\infty$, only the first term contributes while if $d_{P}=d-d_{f}$, then all the fjords are accessible to the projectile and $d_{u}=d_{f}$. They find that the critical dimension for the termite problem is the same as that for the ant problem, $d_{f}=2$. This arises from the fact that for $d_{f}>2$, a random walk is the most efficient way to penetrate another cluster so we choose $d_{f}=2$ in (18). However for $d_{f}<2$, one cluster can penetrate another more efficiently than a random walk and one must set $d_{P}=d_{f}$ in (14).

\section{SUMMARY AND OUTLOOK}

In this brief introduction, we have seen that many physical phenomena of interest may be expressed directly in terms of fractal dimensions. An example that perhaps best illustrates the utility of this correspondence is the superconducting network, where we found that all the physics is given by the unscreened perimeter: $\tilde{\zeta}_{s}=d_{u}[$ Eq. (17)].

Before concluding, we should perhaps emphasize that many of the concepts discussed above apply to a richer range of phenomena than indicated. One example is diffusive annihilation: suppose our ants are particularly hostile to each other and have the property that if they collide 
then they annihilate. If these hostile ants are initially placed at random on a fractal, then their concentration will decay in time with a slow power law tail, whose exponent is given by the ratio $d_{f} / d_{w}{ }^{(47,48)}$

In summary, we have introduced nine different fractal dimensions: $d_{f}=y_{h}, d_{\mathrm{red}}=y_{T}, d_{f}^{\mathrm{BB}}, d_{w}, d_{w}^{\mathrm{BB}}, d_{G}, d_{\min }, d_{E}$, and $d_{u}$. Toulouse has suggested that we call such fractal dimensions "extrinsic," and use the term "intrinsic" to describe exponents such as the chemical distance dimension $d_{l}=d_{f} / d_{\min }$ and the spectral (fracton) dimension $d_{s}=d_{f} / d_{w}$. Like the 26 critical exponents of the early $1960 \mathrm{~s}$, these nine fractal dimensions are probably not all independent-indeed, we have just presented some (but probably not all) of the relations among them. What is the minimum number of fractal dimensions needed to describe the wide range of critical phenomena? This is the analog of the questions that Widom and Kadanoff must have asked. Let us hope that by the time of our next conference someone has been as successful as they were in reducing this "constellation of disorder" to a simple set of principles.

\section{NOTE ADDED IN PROOF}

After this was written, there have been many articles on fractal models of growth that would have been discussed had they appeared slightly earlier. In an effort to correct for this omission, I shall conclude with citations of the original sources so that the interested reader can be brought up to date. ${ }^{(49-59)}$ It should be noted that an increasing effort has been directed toward probing fractal materials experimentally.

\section{REFERENCES}

1. H. E. Stanley, Introduction to Phase Transitions and Critical Phenomena, 2nd ed. (Oxford University Press, New York, 1985). See also H. E. Stanley, Fractal Concepts in Statistical Physics (scheduled for completion December 1984).

2. B. Mandelbrot, Les objects fractals (Flammarion, Paris, 1975) [English versions: Fractals: Form, Chance and Dimension (W. H. Freeman, San Francisco, 1977); The Fractal Geometary of Nature (W. H. Freeman, San Francisco, 1977)].

3. (a) V. Privman, F. Family, and A. Margolina, J. Phys. A, to be published. (b) I. Majid and H. E. Stanley, J. Phys. A, to be published.

4. D. Stauffer, Phys. Rep. 54:1 (1979).

5. F. Family and D. P. Landau, eds., Kinetics of Aggregation and Gelation (North-Holland, Amsterdam, 1984).

6. A. Kapitulnik and G. Deutscher, Phys. Rev. Lett. 43:1444 (1982).

7. R. F. Voss, R. B. Laibowitz, and E. I. Allessandrini, Phys. Rev. Lett. 49:1441 (1982).

8. P. G. de Gennes, La Recherche 7:919 (1976).

9. R. Zallen, The Physics of Amorphous Solids (John Wiley and Sons, New York, 1983).

10. A. Ritzenberg and R. J. Cohen, Phys. Rev. B 30, in press.

11. H. E. Stanley, in Disordered Systems and Localization, C. Castellani, C. DiCastro and L. Peliti, eds. (Springer, Berlin, 1981), p. 59. 
12. B. Jouhier, C. Allain, B. Gauthier-Manuel, and E. Guyon, in Percolation Structures and Processes, G. Deutscher, R. Zallen, and J. Adler, eds. (Adam Hilger, Bristol, 1983), p. 167.

13. G. S. Grest and M. H. Cohen, in Percolation Structures and Processes, G. Deutscher, R. Zallen, and J. Adler, eds. (Adam Hilger, Bristol, 1983), p. 187.

14. L. S. Schulman and P. E. Seiden, in Percolation Structures and Processes, G. Deutscher, R. Zallen, and J. Adler, eds. (Adam Hilger, Bristol, 1983), p. 251.

15. G. Deutscher, A. Kapitulnik, and M. Rappaport, in Percolation Structures and Processes,

G. Deutscher, R. Zallen, and J. Adler, eds. (Adam Hilger, Bristol, 1983), p. 207.

16. D. W. Schaefer and K. D. Keefer, Phys. Rev. Lett., in press.

17. D. W. Schaefer, J. E. Martin, P. Wiltzius, and D. S. Cannell, Phys. Rev. Lett. 52:2371 (1984).

18. D. W. Schaefer, in Kinetics of Aggregation and Gelation (North-Holland, Amsterdam, 1984).

19. C. D. Mitescu and J. Roussenq, in Percolation Structures and Processes, G. Deutscher,

R. Zallen, and J. Adler, eds. (Adam Hilger, Bristol, 1983).

20. H. Nencka-Ficek, $J$. Phys. $A$, to be published.

21. H. E. Stanley, J. Phys. A 10:L211 (1977), Section 4.

22. D. R. Hamann, Phys. Today 36(5):25 (1983).

23. (a) A. Coniglio, J. Phys. A 15:3829 (1982); (b) A. Coniglio, Phys. Rev. Lett. 46:250 (1981); (c) R. Pike and H. E. Stanley, J. Phys. A 14:L169 (1981); (d) D. C. Hong and H. E. Stanley, J. Phys. A 16:L525 (1983); (e) M. F. Sykes, J. Phys. A 17:1565 (1984).

24. H. J. Herrmann and H. E. Stanley, Phys. Rev. Lett. 53:1121 (1984).

25. A. Alexander and R. Orbach, J. Phys. (Paris) Lett. 43:L625 (1982).

26. D. Ben-Avraham and S. Havlin, J. Phys. A 15:L691 (1982).

27. Y. Gefen, A. Aharony, and S. Alexander, Phys. Rev. Lett. 50:77 (1983).

28. R. Rammal and G. Toulouse, J. Phys. (Paris) Lett. 44:L13 (1983).

29. R. Pandey and D. Stauffer, Phys. Rev, Lett. 51:527 (1983); R. Pandey, D. Stauffer, A. Margolina, and J. G. Zabolitzky, J. Stat. Phys. 34:427 (1984).

30. P. Meakin and H. E. Stanley, Phys. Rev. Lett. $51: 1457$ (1983).

31. F. Leyvraz and H. E. Stanley, Phys. Rev. Lett. 51:2048 (1983).

32. H. E. Stanley and A. Coniglio, Phys. Rev. B (RC) 29:522 (1984).

33. A. Aharony and D. Stauffer, Phys. Rev. Lett. 52:2368 (1984).

34. A. Coniglio and H. E. Stanley, Phys. Rev. Lett. 52:1068 (1984).

35. P. Meakin, F. Leyvraz, and H. E. Stanley, Phys. Rev. B, to be published.

36. R. Pike and H. E. Stanley, J. Phys. A 14:L169 (1981).

37. D. C. Hong and H. E. Stanley, J. Phys. A 16:L525; J. Phys. A 16:L525 (1983).

38. A. Coniglio, J. Phys. A 15:3829 (1982).

39. S. Havlin and R. Nossal, J. Phys. A 17:L427 (1984).

40. I. Majid, D. Ben-Avraham, S. Havlin, and H. E. Stanley, Phys. Rev, B 30:1626 (1984).

41. Z. Djordjevic, S. Havlin, H. E. Stanley, and G. H. Weiss, Phys. Rev. B 30:478 (1984).

42. S. Havlin, Z. Djordjevic, I. Majid, H. E. Stanley, and G. H. Weiss, Phys. Rev. Lett. 53:178 (1984).

43. S. Havlin, B. Trus, and H. E. Stanley, Phys. Rev. Lett. 53:1288 (1984).

44. J. Vannimenus, J. P. Nadal, and C. Martin, J. Phys. A 17:L351 (1984).

45. H. J. Herrmann, D. C. Hong, and H. E. Stanley, J. Phys. A 17:L261 (1984).

46. P. G. de Gennes, J. Phys. (Paris) Colloque 41:C3 (1980).

47. P. Meakin and H. E. Stanley, J. Phys. A 17:L173 (1984).

48. K. Kang and S. Redner, Phys. Rev. Lett. 52:955 (1984).

49. C. Allain and B. Gauthier-Manuel, Phys. Rev. Lett., in press. 
50. H. D. Bale and P. W. Schmidt, Phys. Rev. Lett. 53:596 (1984).

51. H. G. E. Hentschel, Phys. Rev. Lett. 52:212 (1984).

52. H. G. E. Hentschel and J. Deutch, Phys. Rev. A 29:1609 (1984).

53. R. B. Laibowitz and Y. Gefen, Phys. Rev. Lett. 53:380 (1984).

54. J. E. Martin and B. W. Ackerson, Phys. Rev. Lett., in press.

55. M. Matsushita, M. Sano, Y. Hayakawa, H. Honjo, and Y. Sawada, Phys. Rev. Lett. 53:286 (1984).

56. M. Muthukumar, Phys. Rev. Lett. 50:839 (1983).

57. L. Niemeyer, L. Pietronero, H. J. Weismann, Phys. Rev. Lett. 52:1033 (1984).

58. M. Tokuyama and K. Kawasaki, Phys. Lett. 100A:337 (1984).

59. D. A. Weitz and M. Oliveira, Phys. Rev. Lett. 52:1433 (1984). 